\title{
Inducible transgenic expression in the short-lived fish Nothobranchius furzeri
}

\author{
J. B. Allard, H. Kamei and C. Duan* \\ Department of Molecular, Cellular and Developmental Biology, University of Michigan, \\ Natural Science Building, 830 N. University Avenue, Ann Arbor, MI 48109-1048, U.S.A.
}

(Received 28 July 2012, Accepted 13 February 2013)

\begin{abstract}
This study demonstrates inducible transgenic expression in the exceptionally short-lived turquoise killifish Nothobranchius furzeri, which is a useful vertebrate model for ageing research. Transgenic $N$. furzeri bearing a green fluorescent protein (Gfp) containing construct under the control of a heat shock protein 70 promoter were generated, heat shock-induced and reversible Gfp expression was demonstrated and germline transmission of the transgene to the F1 and F2 generations was achieved. The availability of this inducible transgenic expression system will make the study of ageing-related antagonistically pleiotropic genes possible using this unique vertebrate model organism.

(C) 2013 The Authors

Journal of Fish Biology (c) 2013 The Fisheries Society of the British Isles
\end{abstract}

Key words: ageing; heat shock protein 70 promoter; transgenic fish; turquoise killifish.

Short-lived genetically tractable model systems have been extremely useful for ageing research. It remains challenging, however, to extend findings from invertebrate models such as the nematode Caenorhabditis elegans and fruit fly Drosophila melanogaster to vertebrates. This is in part because the major vertebrate models for ageing research, mice Mus musculus and zebrafish Danio rerio (Hamilton, 1882), are comparatively long-lived and expensive to work with (Allard \& Duan, 2011). There is a need for a short-lived vertebrate model that bridges the gap between flies and mice, and one animal that has been proposed to fill this role is the turquoise killifish Nothobranchius furzeri Jubb 1971 (Genade et al., 2005).

Nothobranchius furzeri is an annual fish that inhabits transient seasonal ponds. Each year during the dry season, the ponds in which they live dry up completely. The adult fish die when this happens but their eggs are adapted to survive desiccation and are able to remain in a state of developmental diapause in the soil until the next rainy season when they hatch. The natural adult lifespan of these fish is therefore limited to $<1$ year in most cases, and indeed, even in captivity, these fish live only for several months, with the shortest-lived strain living only for $c .12$ weeks (Valdesalici \& Cellerino, 2003; Terzibasi et al., 2008). Because of their short natural

*Author to whom correspondence should be addressed. Tel.: +1 734763 4710; email: cduan@umich.edu 
lifespan, small size, low cost of maintenance and the ability of their embryos to be stored for periods of a year or more in a state of diapause, this species is being developed as a new model for ageing research (Genade et al., 2005). Recent studies in $N$. furzeri have shown that its ageing is accompanied by the appearance of cellular biomarkers of ageing (such as lipofuscin and senescence-associated $\beta$-galactosidase), pathological changes that are observed with age in other teleosts (such as spontaneous neoplasias) and a variety of ageing-related degradations in heart, liver, kidney, gonad and vascular tissues (Genade et al., 2005; Di Cicco et al., 2011). In addition, caloric restriction, a reduction in temperature and treatment with the small molecule resveratrol were shown to extend lifespan in $N$. furzeri (Valenzano et al., 2006a, b; Terzibasi et al., 2008). An initial characterization of the $N$. furzeri genome has also been published (Reichwald et al., 2009).

Despite many of its advantages as a promising model organism in ageing biology, the $N$. furzeri model has not been widely used. This was in part because tools for genetic manipulation in $N$. furzeri were very limited. The aim of this study was to develop stable and inducible transgenic expression in this species. While two very recent studies have demonstrated the successful generation of transgenic lines in $N$. furzeri (Valenzano et al., 2011; Hartmann \& Englert, 2012), inducible transgenic expression was not successful.

In order to facilitate the incorporation of injected DNA into the N. furzeri genome, the Tol-2 transposon from the medaka Oryzias latipes (Temminck \& Schlegel 1846) was employed. An autonomous Tol-2 element was discovered in O. latipes that contains a gene encoding a functional transposase, which has been shown to catalyse transposition even in other species (Kawakami et al., 1998, 2004; Kawakami \& Noda, 2004). A Tol-2 construct was utilized (pT2AL200R150G) in which the transposase gene has been replaced with a green fluorescent protein (Gfp) gene ( $g f p$ ) (Urasaki et al., 2006). When this construct is microinjected into embryos at the one-cell stage along with mRNA encoding the transposase (Kawakami \& Shima, 1999) the gfp gene should be transposed into the genome.

Using this approach, it was possible to generate Gfp-positive $N$. furzeri. Eggs for microinjection were obtained by natural cross of wild-type $N$. furzeri adults using fine sand as a mating substratum and were collected within $1 \mathrm{~h}$. The eggs were aligned and immobilized within a $1 \mathrm{~mm}$ wide furrow in an agarose dish. Injection solutions were prepared with Tol-2 plasmid DNA at a concentration of $100 \mathrm{ng} \mu \mathrm{l}^{-1}$, Tol2 transposase messenger (m)RNA at a concentration of $100 \mathrm{ng}^{-1} \mathrm{l}^{-1}$ and $0 \cdot 1 \%$ phenol red dye. Approximately, $100 \mathrm{pg}$ DNA and $100 \mathrm{pg}$ mRNA were injected into each egg at the boundary between the yolk and the cell at the one-cell stage [Fig. 1(a)].

Overall, of the 433 total eggs injected in 20 independent injection experiments, $189(43 \%)$ survived the injection and were alive on the third day post fertilization. Of these surviving larvae, 39 (20\%) were transgenic [Fig. 1(b)]. In many cases the F0 transgenic founders exhibited strong whole body Gfp expression and in other cases mosaic expression was observed. Three juvenile F0 transgenic fish are shown in Fig. 1(c). The fish at the top exhibits full body Gfp expression. The other two fish exhibit mosaic Gfp expression. Of these F0 transgenic fish, six (16\%) reached adulthood and were crossed with wild-type fish. These F0 fish gave transmission frequencies ranging from 5 to $100 \%$. The fish shown on the top in Fig. 1(c) produced $100 \%$ F1 transgenic progeny. This $100 \%$ transmission efficiency indicates that multiple separate transgene insertions probably took place. The fish in the middle 

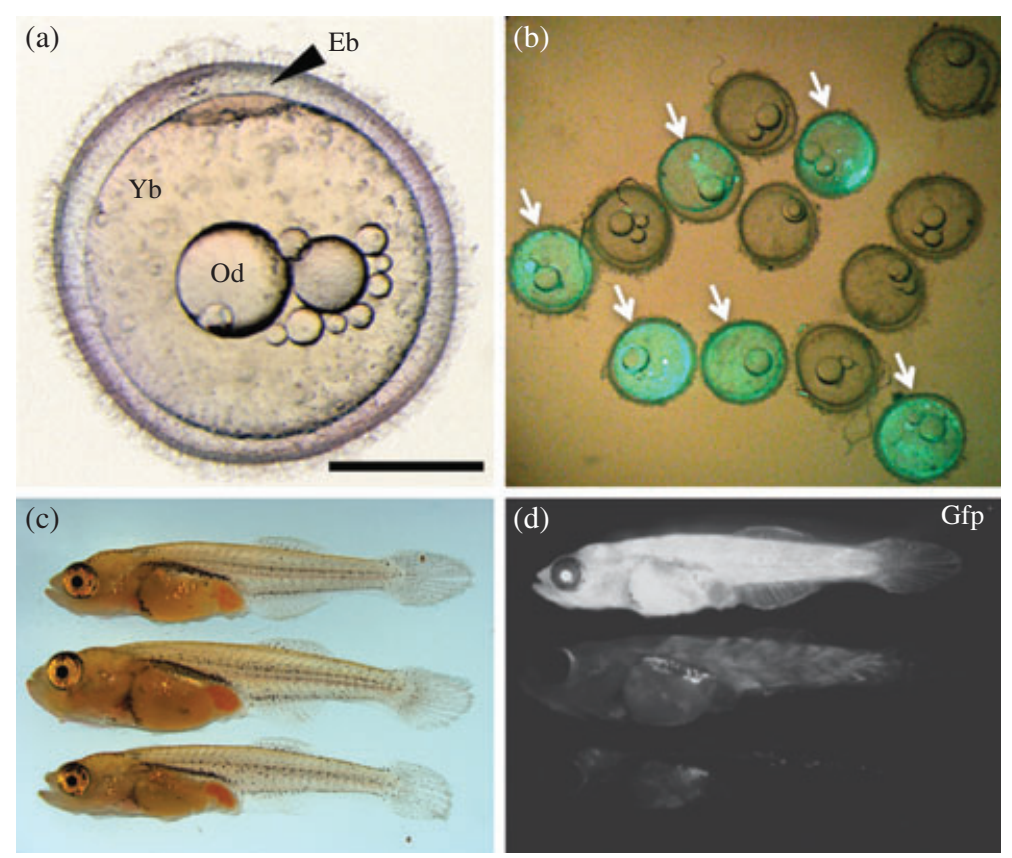

FIG. 1. Development of stable transgenesis in Nothobranchius furzeri. (a) Bright field image of a one-cell stage embryo. ₹, the embryonic body (Eb); yolk body (Yb); oil droplet (Od). Scale bar $=500 \mu \mathrm{m}$. (b) A group of embryos at 4 days post fertilization. They were injected with Tol-2 transposase mRNA and the pT2AL200R150G plasmid DNA. The pT2AL200R150G plasmid contains the green fluorescent protein (Gfp) gene (gfp) flanked by two Tol-2 sites. Expression of $g f p$ is under the control of the constitutive EF1a promoter. Gfp fluorescence is visible in six of 13 eggs, and transgenic eggs are marked $(\rightarrow)$. (c, d) Three juvenile F0 gfp transgenic fish are shown: (c) is the bright field view and (d) is the Gfp fluorescence view. The top fish shows full body Gfp expression. The remaining two fish show varying levels of mosaic Gfp expression.

in Fig. 1(c) produced 196 embryos. Among them, 10 were transgenic, reflecting a $5 \cdot 1 \%$ transgenic efficiency.

In order to demonstrate the feasibility of inducible transgene expression in $N$. furzeri, a Tol-2 vector containing a Gfp-tagged transgene under the control of a $D$. rerio heat shock protein 70 (Hsp70) promoter was utilized (Halloran et al., 2000; Kamei et al., 2011). This promoter allows heat-shock-inducible expression of the transgene in D. rerio (Halloran et al., 2000). Using the transgenesis method described above, transgenic $N$. furzeri that harboured this construct were generated. The fish initially showed no Gfp signal but in response to $1 \mathrm{~h}$ of heat shock at $37^{\circ} \mathrm{C}, g f p$ expression was induced as demonstrated by fluorescence microscopy. The heat shock treatment was administered by placing a 11 tank of room temperature water containing the fish into a larger water bath at $37^{\circ} \mathrm{C}$ and removing it after $1 \mathrm{~h}$. The fish showed no abnormality during or after the treatment. Embryos, juveniles and adult fish were treated in this manner and the treatment did not cause mortality or any other detectable adverse effects. Figure 2(a), (b) shows a juvenile F0 transgenic fish before heat shock. Figure 2(c)-(f) shows the same fish at 1, 3, 9 and $22 \mathrm{~h}$ after the heat shock treatment. Maximum $g f p$ signal was reached at $9 \mathrm{~h}$ and then began 

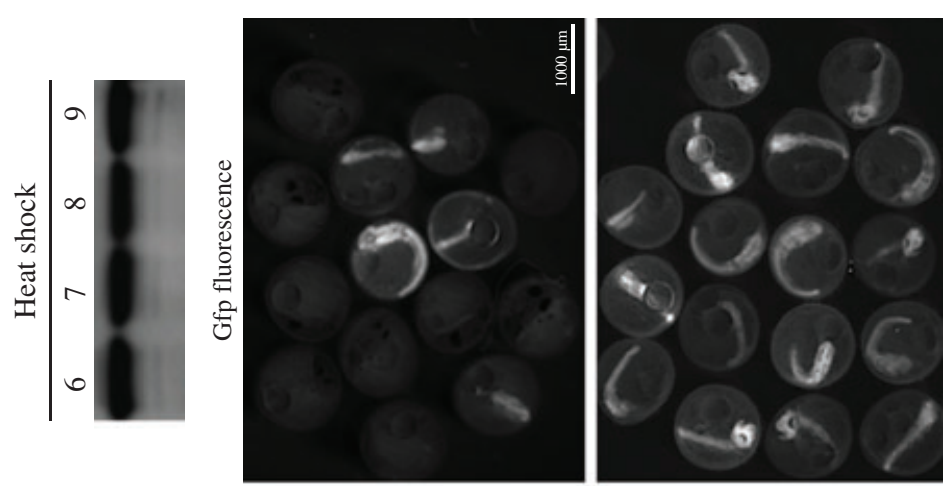

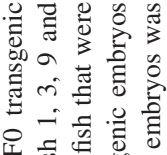

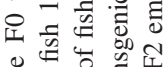

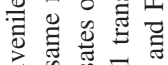

包可届

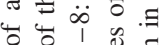

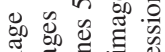

范苛苛

긍 헝 ठ

एँ

क्ष

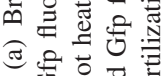

ङ 웜월

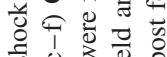

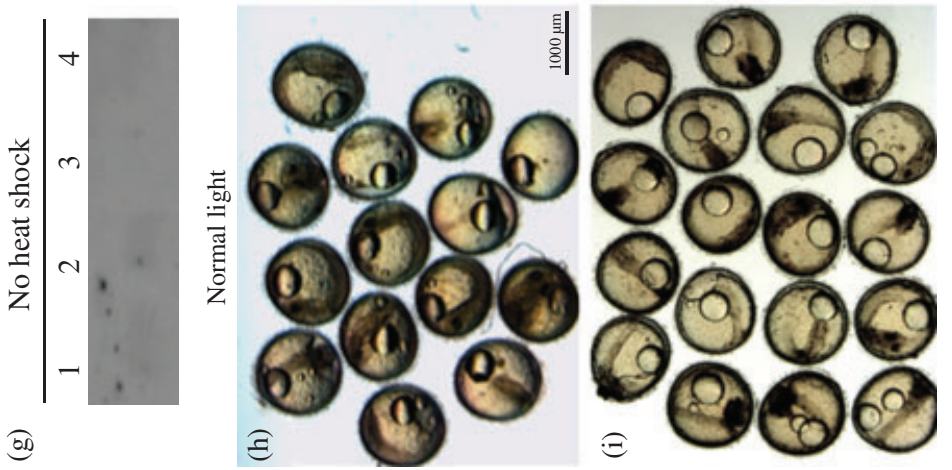

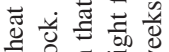

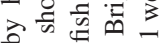

च छ

ज

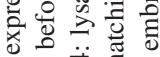

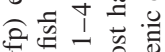

0

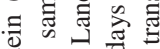

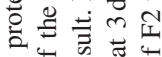

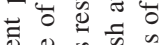

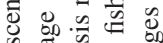

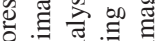

记完

듕 흥 홍

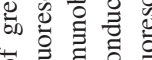

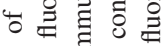

휴융 है

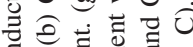

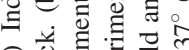

등 吾

क क क त्र

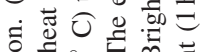

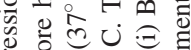

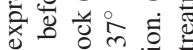

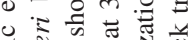

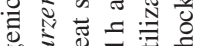

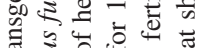

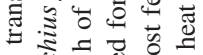

은

을

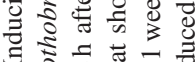

i

乌் 
to fall. Immunoblot with an anti-gfp antibody confirmed that the transgene was not expressed before heat shock, but was induced afterwards [Fig. 2(g)]. It was possible to cross F0 transgenic founders with wild-type fish and obtain F1 transgenic progeny, thus demonstrating germline transmission of the transgene [Fig. 2(h)]. F1 transgenic fish were raised to adulthood and crossed with each other to produce F2 progeny. Many F2 embryos were obtained and 100\% were transgenic [Fig. 2(i)].

This study demonstrates robust inducible expression of $g f p$ in N. furzeri. Although two recent studies by Valenzano et al . (2011) and Hartmann \& Englert (2012) report the successful generation of $g f p$-expressing transgenic $N$. furzeri lines, no inducible transgenic expression has been reported for this species. The study by Valenzano et al. (2011) used a D. rerio Hsp70 promoter appearing to be derived from the same source as this study (Halloran et al., 2000). In their study, however, no heat shock-inducible expression was observed (Valenzano et al., 2011). The construct used in this study was generated by directly subcloning the Hsp70 promoter from the plasmid DNA used in the study by Halloran et al. (2000). The specific sequence of the Hsp70 promoter used in Valenzano et al. (2011) was not stated. The results of this study indicate that it is possible to use a $D$. rerio-inducible promoter in $N$. furzeri. The study by Hartmann \& Englert (2012) used the D. rerio $\beta$-actin 2 gene promoter, which drives broad expression in $N$. furzeri as in $D$. rerio. Therefore, many genetic and molecular tools developed for $D$. rerio and other fish species may be able to be employed in $N$. furzeri .

The antagonistic pleiotropy theory of ageing suggests that many ageing genes may have essential roles in early life or embryonic development (Williams, 1957). Therefore, in many cases, it is necessary to use inducible expression systems that allow temporally specific expression when studying these genes by transgenic methods. The inducible expression technique reported in this study will allow candidate longevity genes to be assessed in adult $N$. furzeri even when their expression in the early life stage may be detrimental.

The authors gratefully acknowledge A. Cellerino for his support and helpful advice regarding the maintenance of an $N$. furzeri colony in the laboratory. This study was supported by NIH Grant 1R21AG040604-01A1 and NSF Grant IOS-1051034 to C.D.

\section{References}

Allard, J. B. \& Duan, C. (2011). Comparative endocrinology of aging and longevity regulation. Frontiers in Endocrinology 2, 75.

Di Cicco, E., Tozzini, E. T., Rossi, G. \& Cellerino, A. (2011). The short-lived annual fish Nothobranchius furzeri shows a typical teleost aging process reinforced by high incidence of age-dependent neoplasias. Experimental Gerontology 46, 249-256.

Genade, T., Benedetti, M., Terzibasi, E., Roncaglia, P., Valenzano, D. R., Cattaneo, A. \& Cellerino, A. (2005). Annual fishes of the genus Nothobranchius as a model system for aging research. Aging Cell 4, 223-233.

Halloran, M. C., Sato-Maeda, M., Warren, J. T., Su, F., Lele, Z., Krone, P. H., Kuwada, J. Y. \& Shoji, W. (2000). Laser-induced gene expression in specific cells of transgenic zebrafish. Development 127, 1953-1960.

Hartmann, N. \& Englert, C. (2012). A microinjection protocol for the generation of transgenic killifish (Species: Nothobranchius furzeri). Developmental Dynamics 241, 1133-1141.

Kamei, H., Ding, Y., Kajimura, S., Wells, M., Chiang, P. \& Duan, C. (2011). Role of IGF signaling in catch-up growth and accelerated temporal development in zebrafish embryos in response to oxygen availability. Development 138, 777-786. 
Kawakami, K. \& Noda, T. (2004). Transposition of the Tol2 element, an Ac-like element from the Japanese medaka fish Oryzias latipes, in mouse embryonic stem cells. Genetics 166, 895-899.

Kawakami, K. \& Shima, A. (1999). Identification of the Tol2 transposase of the medaka fish Oryzias latipes that catalyzes excision of a nonautonomous Tol 2 element in zebrafish Danio rerio. Gene 240, 239-244.

Kawakami, K., Koga, A., Hori, H. \& Shima, A. (1998). Excision of the tol2 transposable element of the medaka fish, Oryzias latipes, in zebrafish, Danio rerio. Gene 225, $17-22$.

Kawakami, K., Imanaka, K., Itoh, M. \& Taira, M. (2004). Excision of the Tol2 transposable element of the medaka fish Oryzias latipes in Xenopus laevis and Xenopus tropicalis. Gene 338, 93-98.

Reichwald, K., Lauber, C., Nanda, I., Kirschner, J., Hartmann, N., Schories, S., Gausmann, U., Taudien, S., Schilhabel, M. B., Szafranski, K., Glockner, G., Schmid, M., Cellerino, A., Schartl, M., Englert, C. \& Platzer, M. (2009). High tandem repeat content in the genome of the short-lived annual fish Nothobranchius furzeri: a new vertebrate model for aging research. Genome Biology 10, R16.

Terzibasi, E., Valenzano, D. R., Benedetti, M., Roncaglia, P., Cattaneo, A., Domenici, L. \& Cellerino, A. (2008). Large differences in aging phenotype between strains of the short-lived annual fish Nothobranchius furzeri. PLoS One 3, e3866.

Urasaki, A., Morvan, G. \& Kawakami, K. (2006). Functional dissection of the Tol2 transposable element identified the minimal cis-sequence and a highly repetitive sequence in the subterminal region essential for transposition. Genetics 174, 639-649.

Valdesalici, S. \& Cellerino, A. (2003). Extremely short lifespan in the annual fish Nothobranchius furzeri. Proceedings of the Royal Society B 270(Suppl. 2), S189-S191.

Valenzano, D. R., Terzibasi, E., Cattaneo, A., Domenici, L. \& Cellerino, A. (2006a). Temperature affects longevity and age-related locomotor and cognitive decay in the short-lived fish Nothobranchius furzeri. Aging Cell 5, 275-278.

Valenzano, D. R., Terzibasi, E., Genade, T., Cattaneo, A., Domenici, L. \& Cellerino, A. (2006b). Resveratrol prolongs lifespan and retards the onset of age-related markers in a short-lived vertebrate. Current Biology 16, 296-300.

Valenzano, D. R., Sharp, S. \& Brunet, A. (2011). Transposon-mediated transgenesis in the short-lived African killifish Nothobranchius furzeri, a vertebrate model for aging. G3: Genes, Genomes, Genetics 1, 531-538.

Williams, G. C. (1957). Pleiotropy, natural selection, and the evolution of senescence. Evolution 11, 398-411. 